\title{
A Rare Case of Clostridium septicum Causing an Abdominal Aortic Endograft Leak
}

\author{
Amanda Kravetz ${ }^{\mathrm{a}}$, Sadie Martin ${ }^{\mathrm{a}}$, Gregory Fortin ${ }^{\mathrm{b}}$, Umer Feroze Malik ${ }^{\mathrm{c}, \mathrm{d}}$
}

\begin{abstract}
The authors report a case of an African American female, status post-EVAR one and a half years prior, who presented to the emergency department with sudden onset of abdominal pain. Computed tomography revealed a type I endoleak and the presence of periaortic gas. The patient underwent an emergent open abdominal aortic endograft explant with in situ tube graft replacement. Blood and endograft tissue cultures grew Clostridium septicum. The patient died perioperatively of systemic multiple organ failure. C. septicum is a rare infection with lethal propensities and has a strong association with paraneoplastic syndromes. The underlying etiology of this patient's bacteremia was likely associated with an immunodeficiency secondary to myelodysplastic syndrome. Importantly, if periaortic gas is present, the disease is far advanced and the prognosis is poor regardless of treatment; however, a combination of antibiotics and surgery should be initiated immediately.
\end{abstract}

Keywords: Clostridium septicum; Abdominal aortic endograft leak; Myelodysplastic syndrome

\section{Introduction}

We present the only case report in which an abdominal aortic endograft has been infected with Clostridium septicum in the presence of documented myelodysplastic syndrome. Clostridium septicum has been associated with a variety of malignancies, particularly gastrointestinal and hematologic malignancies; however, only $8 \%$ of $C$. septicum infections are vascular-related. To our knowledge, there are only 31 reported cases of $C$. septicum causing aortic infection in the English literature, and this case represents the third report of post-endovascular aortic aneurysm repair (EVAR) stent graft infection with C. septicum.

\section{Case Report}

An 81-year-old African American female presented to the emergency department with a 2-h presentation of sudden onset of severe bilateral lower leg, back, and localized periumbilical abdominal pain. She previously underwent an infrarenal EVAR with bilateral iliac artery limb extension as well as pacemaker insertion approximately 1.5 years prior. Past medical history consisted of myelodysplastic syndrome with chronic thrombocytopenia (platelet count normally around 40,000), COPD with home oxygen usage, hypertension, atrial fibrillation, insulin-dependent diabetes mellitus, endocarditis, and history of myocardial infarction.

On admission, vital signs revealed a blood pressure of $82 / 50$, heart rate of 100 beats per minute, respiratory rate of 16 , temperature of $36^{\circ} \mathrm{C}$, and pulse oximetry of $95 \%$ on $3 \mathrm{~L}$ nasal cannula. Physical examination revealed the patient to be in moderate shortness of breath at rest. She appeared pale and restless, yet she was able to give an adequate history. Her abdomen was soft, non-tender, and non-distended. She

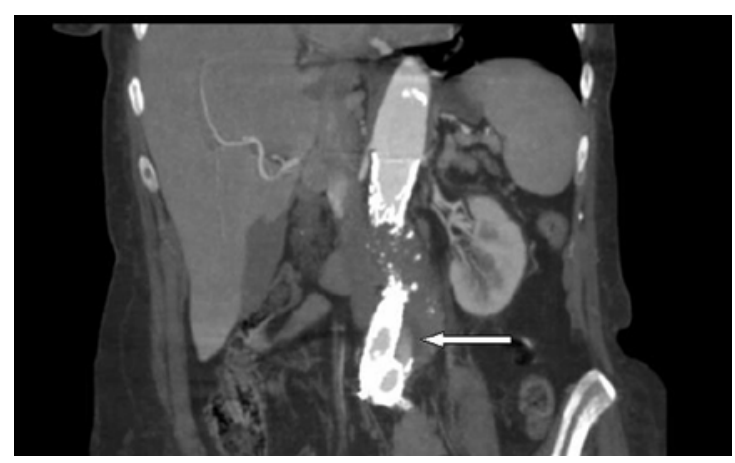

Figure 1. Coronal view: abdominal aortic endograft leak. 


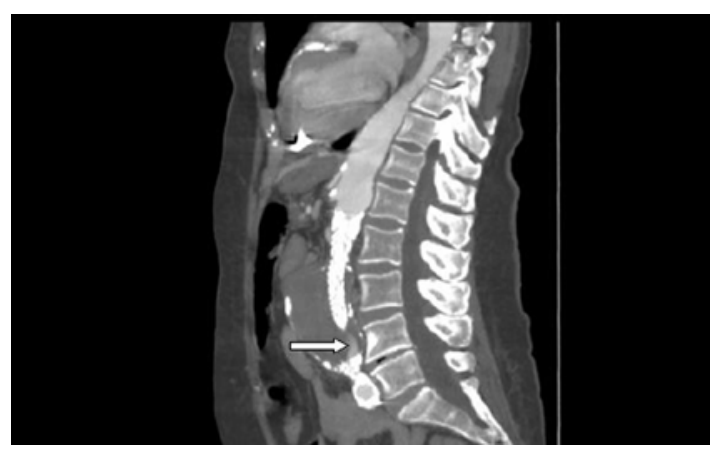

Figure 2. Sagittal view: abdominal aortic endograft leak.

had palpable pulses distally and no cyanosis or edema in her lower extremities.

The patient was admitted to the intensive care unit, and initial treatment consisted of fluid resuscitation, morphine, and piperacillin-tazobactam. After $2 \mathrm{~L}$ of normal saline (NS), she remained hypotensive. Dopamine infusion was then started at $5 \mathrm{mcg} / \mathrm{kg} / \mathrm{min}$. The patient appeared to have decreased respiratory effort and became more lethargic. Endotracheal intubation was performed and a subclavian central venous catheter was inserted. Dopamine infusion was increased to $10 \mathrm{mcg} / \mathrm{kg} / \mathrm{min}$, and a third liter of NS was given.

Laboratory data revealed an elevated white blood cell count of 20,100 with $80 \%$ neutrophils and 3\% bands. Hemoglobin measured $10.8 \mathrm{~g} / \mathrm{dL}$, serum lactate was 7.9 , and the platelet count was 5,000. A complete metabolic panel, amylase, lipase, cardiac enzymes, and a urine analysis were all within normal limits.

A computed tomographic (CT) angiogram of the chest, abdomen, and pelvis revealed an enlarging saccular infrarenal native aneurysm sac of $6.1 \times 5.6 \mathrm{~cm}$ compared to a previous scan 2 months prior, which measured $5.8 \times 5.3 \mathrm{~cm}$. A type I endoleak appeared to arise from the left limb of the endograft (Fig. 1,2) where the native aorta was noted to be emphysematous at the anterior margin of the native capsule (Fig. 3), consistent with clostridial infection.

Immediately after the CT scan was completed, the vascular surgery team was notified and the patient was taken to the operating room. The retroperitoneum was explored through an anterior midline incision. There was no visible purulence, hematoma or active bleeding surrounding the native aneurysm; however, the tissues were extremely hyperemic and weak. The native aneurysm did appear to have contained the small leak as seen on the CT scan. The endograft was explanted. A $16 \mathrm{~mm}$ Dacron graft was cut to appropriate size and sutured end-to-end from the infrarenal aorta to the origin of the common iliac arteries. The patient was given 4 units of blood, 4 units of fresh frozen plasma, and 18 units of platelets intraoperatively.

The operation was completed without complication and the patient was then transferred to the ICU in severe septic

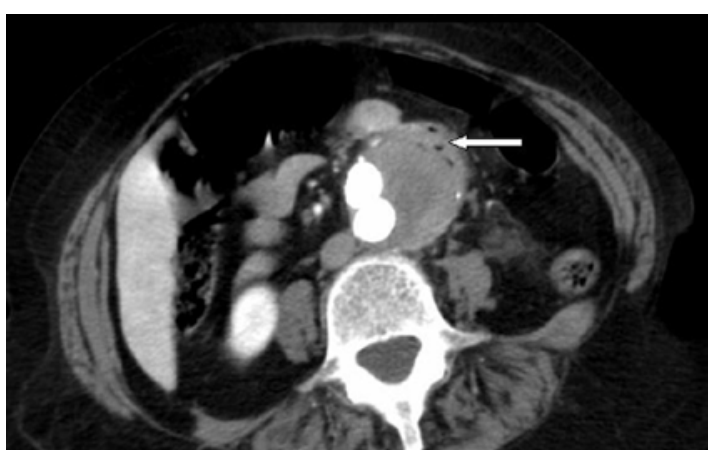

Figure 3. Axial view: periaortic gas within the anterior capsule of native aneurysm.

shock. Blood and tissue cultures were positive for C. septicum. The patient was seen by infectious disease immediately after her surgery, and clindamycin and metronidazole therapies were also instituted. Despite all resuscitative efforts, the patient's postoperative course was complicated by systemic multiple organ failure and she expired $36 \mathrm{~h}$ after surgery.

\section{Discussion}

C. septicum is a Gram-positive, highly motile bacillus that rarely causes bacteremia, and because of their fastidious nature, these organisms are often difficult to isolate from infected sites [1]. The mortality rate of $C$. septicum bacteremia is between 33 and $64 \%$, whereas the mortality rate associated with tissue infection is approximately $70-100 \%$ [1-3]. C. septicum is believed to cause less than $1.3 \%$ of all clostridial infections [4]. To our knowledge, only 31 reported cases of $C$. septicum causing aortic infection exist in the English literature [3, 5-7] and of those, only four cases report a concomitant myelodysplastic syndrome and a $C$. septicum bloodstream-related infection [8], and none of these patients had a documented aortitis.

Hermsen et al extensively researched PubMed in 2008 [8] evaluating infectious phenotypes and survival among 231 patients with $C$. septicum and found that the vascular phenotype occurred in only $8 \%$ of the patients with a $68 \%$ survival rate. Their extensive review also noted that patients with a C. septicum vascular infection had an $80 \%$ survival rate with surgery compared to $25 \%$ survival rate with without surgery, a statistically significant finding. Of the seven anatomically based phenotypes, the most common was non-traumatic infection of the skin and soft tissues (52\%), secondly, bacteremia $(23 \%)$, and the remaining were vascular, intraperitoneal, central nervous system, musculoskeletal, and head and neck infections. Of note, although they discovered diabetes to be a risk factor for development of a skin and soft tissue C. septicum infection, they did not note a difference in survival between diabetics and non-diabetics. 
C. septicum has been associated with a variety of malignancies, particularly gastrointestinal or hematologic malignancies $[1,3,8,9]$. Hermsen et al found that $C$. septicum infection occurs in only $20 \%$ of patients without known or occult malignancies. Despite a widely held belief that $C$. septicum is part of normal gastrointestinal flora, a previous study which examined the stool of healthy individuals only isolated C. septicum in one individual [10]. Nonetheless, when isolated in a patient with systemic illness, it generally has been known to cause rapid progressive soft tissue infection and myonecrosis $[4,8,11]$.

The pathophysiology of $C$. septicum to proliferate in ill patients is not entirely clear. The mechanism of pathogen propagation has been shown in tissues with low $\mathrm{pH}$ and oxygen levels [1]. Clostridial spores arise with arterial injury and flourish in tissues associated with necrosis, ischemia, anoxia, and increased lactic acid levels $[12,13]$. A fairly accepted postulation that explains the association of $C$. septicum with gastrointestinal malignancies has to due with the disruption of the mucosal surface, which may serve as a portal for clostridial organisms to enter the bloodstream and seed at atherosclerotic junctures [1, 3, 14]. Furthermore, hematologic diseases compromise the immune system and may cause thrombocytopenia and neutropenia, thus altering the endothelial tissues, preventing barrier protection [13]. Hence, in our case, the patient was not only immunocompromised but she also had previous tissue disruption from her EVAR surgery, which we believe made her more susceptible to the portal entry of anaerobic organisms. To note, this case is only the third report of post-EVAR stent graft infection involving $C$. septicum $[15,16]$. The rate of infection with EVAR is unknown. Review of the literature demonstrates an overall incidence of prosthetic aortic graft infection to be approximately $0.43-1.17 \%$ [16], thus leaving the rate of EVAR infection to be even less.

Gas formation surrounding the aorta or peripheral arteries is a typical presentation of clostridial infection and emergent surgical treatment is the current consensus to achieve optimal results $[8,14,17]$. Sailors et al found that 7 out of 8 patients with intramural gas involving the arterial wall died as a result of arterial C. septicum infection, which is a sign of poor prognosis. Thus, a lack of emergent and direct adequate therapy against these organisms is more likely to result in death.

Surgical treatment options include debridement of the infected arterial segments and extra-anatomic revascularization with an axillary bifemoral bypass versus in situ graft replacement. We chose a midline abdominal approach to explore the aneurysm sac and to evaluate for possible leak or rupture, followed by replacement of the endograft with an in situ Dacron tube graft. In support of undergoing surgery, Seder et al revealed a 75\% 6-month survival rate in patients treated with in situ grafting (Dacron, polyethylene terephtahalte, or polytetrafluoroethylene) after C. septicum aortic infection, and a $100 \%$ mortality within 6 months if surgical treatment was not instituted as part of the therapy.

It has been documented that once $C$. septicum is identified in arteriosclerotic lesions, it cannot be eradicated with antibiotic therapy alone [18]. However, antibiotics should be administered immediately, and this has shown to consistently improve survival [3]. Initially, broad-spectrum antibiotics should be administered. The most effective are clindamycin, metronidazole, chloramphenicol, cefoxitin, a combination of penicillin plus a beta-lactamase inhibitor, tigecycline, moxifloxacin, and the carbapenems [14]. In our case, we used a combination of clindamycin, metronidazole, and piperacillin/tazobactam, which were ideal for this patient despite her outcome. If she had lived, life-long antibiotic therapy would have been considered.

\section{Conclusion}

Presumptive identification of $C$. septicum should be based on awareness of periaortic gas seen on the preoperative CT scan, Gram stain during surgery, and awareness of C. septicum as a paraneoplastic syndrome. As a differential diagnosis, the definitive therapy should be immediate antibiotics and surgery. Furthermore, if periaortic gas is present, then the disease is far advanced and the prognosis is poor regardless of treatment.

Due to the low number of reported cases and limited information regarding myelodysplastic syndrome and $C$. septicum, meaningful comparisons are not possible. However, this case report reaffirms the relationship of C. septicum infection with active immunosuppression and is only the third case report of post-EVAR stent graft infection with C. septicum.

\section{References}

1. Sailors DM, Eidt JF, Gagne PJ, Barnes RW, Barone GW, McFarland DR. Primary Clostridium septicum aortitis: a rare cause of necrotizing suprarenal aortic infection. A case report and review of the literature. J Vasc Surg. 1996;23(4):714-718.

2. Pelletier JP, Plumbley JA, Rouse EA, Cina SJ. The role of Clostridium septicum in paraneoplastic sepsis. Arch Pathol Lab Med. 2000;124(3):353-356.

3. Seder CW, Kramer M, Long G, Uzieblo MR, Shanley CJ, Bove P. Clostridium septicum aortitis: Report of two cases and review of the literature. J Vasc Surg. 2009;49(5):1304-1309.

4. Gorbach SL, Thadepalli H. Isolation of Clostridium in human infections: evaluation of 114 cases. J Infect Dis. 1975;131(Suppl):S81-85.

5. Granier M, Granier A, Fraga J, Durant R. Emphysematous infectious aortitis: a dramatic evolution. Eur Heart 


\section{J. 2011;32(16):2085.}

6. Moseley B, Mwirigi NW, Bowen J. Clostridium septicum Aortitis and Cecal Adenocarcinoma. Case Rep Med. 2010;2010:121728.

7. Yang Z, Reilly SD. Clostridium septicum aortitis causing aortic dissection in a 22-year-old man. Tex Heart Inst J. 2009;36(4):334-336.

8. Hermsen JL, Schurr MJ, Kudsk KA, Faucher LD. Phenotyping Clostridium septicum infection: a surgeon's infectious disease. J Surg Res. 2008;148(1):67-76.

9. Pouwels MJ, Donnelly JP, Raemaekers JM, Verweij PE, de Pauw BE. Clostridium septicum sepsis and neutropenic enterocolitis in a patient treated with intensive chemotherapy for acute myeloid leukemia. Ann Hematol. 1997;74(3):143-147.

10. Finegold SM, Attebery HR, Sutter VL. Effect of diet on human fecal flora: comparison of Japanese and American diets. Am J Clin Nutr. 1974;27(12):1456-1469.

11. Maclennan JD. The histotoxic clostridial infections of man. Bacteriol Rev. 1962;26:177-276.

12. Malmgren RA, Flanigan CC. Localization of the vegetative form of Clostridium tetani in mouse tumors following intravenous spore administration. Cancer Res.
1955;15(7):473-478.

13. Thiele EH, Arison RN, Boxer GE. Oncolysis by Clostridia. Iv. Effect of Nonpathogenic Clostridial Spores in Normal and Pathological Tissues. Cancer Res. 1964;24:234-238.

14. Brook I. Anaerobic bacteria as a cause of mycotic aneurysm of the aorta: microbiology and antimicrobial therapy. Curr Cardiol Rev. 2009;5(1):36-39.

15. Halak M, Heldenberg E, Silverberg D, Schneiderman J. Clostridium septicum post-endovascular aneurysm repair stent-graft infection. Vascular. 2012;20(2):104-106.

16. Colwick SE, Alkhoury F, Martin JT, Ferneini AM. Abdominal aortic aneurysm repair complicated by infection with Clostridium septicum. Ann Vasc Surg. 2011;25(6):839 e811-833.

17. Takano H, Taniguchi K, Kuki S, Nakamura T, Miyagawa $\mathrm{S}$, Masai T. Mycotic aneurysm of the infrarenal abdominal aorta infected by Clostridium septicum: a case report of surgical management and review of the literature. J Vasc Surg. 2003;38(4):847-851.

18. Liechti ME, Schob O, Kacl GM, Caduff B. Clostridium septicum aortitis in a patient with colon carcinoma. Eur J Clin Microbiol Infect Dis. 2003;22(10):632-634. 